\title{
First-principle study of geometric stabilities, electronic and magnetic properties of low coverage vanadium adsorption on graphene
}

\begin{abstract}
Stable geometries, electronic and magnetic properties of low coverage vanadium (V) atoms adsorption on graphene sheet have been investigated by first principles calculations, using generalized gradient approximation. Calculation shows that center of the ring is energetically favorable for both $\mathrm{V}$ adatom and perpendicular dimer after relaxation. Moreover, the proportion of orbital contribution of $\mathrm{C}-\mathrm{V}$ bonding are mainly dominated by $2 \mathrm{pz}$ orbital of $\mathrm{C}$ and partially occupied by the $3 \mathrm{~d}$ like states of V. It is also found that the low coverage $\mathrm{V}$ atom adsorbed graphene system is metallic and magnetic, and has demonstrated additional hint on its usefulness in magnetic devices.
\end{abstract}

Keyword: Electric and magnetic properties; Geometric; Graphene 